\title{
Functional and Partial Morphological Regression of Established Renal Injury in the Obese Zucker Rat by Blockade of the Renin-Angiotensin System
}

\author{
Katarína Šebekováa Maria Lill $^{\mathrm{b}}$ Peter Boor $^{\mathrm{a}} \quad$ August Heidland $^{\mathrm{c}}$ \\ Kerstin Amann ${ }^{b}$ \\ a Department of Clinical and Experimental Pharmacotherapy, Slovak Medical University, Bratislava, Slovakia; \\ ${ }^{b}$ Department of Pathology, University of Erlangen-Nuremberg, Erlangen, and ' Department of Internal Medicine, \\ University of Würzburg, Würzburg, Germany
}

\section{Key Words}

Obese Zucker rat • Glomerulosclerosis · Tubulointerstitial fibrosis · Glomerular cell number • Proteinuria •

Angiotensin-converting enzyme inhibitors · Angiotensin II receptor 1 blockers

\begin{abstract}
Background/Aims: In experimental nephropathies, inhibitors of the renin-angiotensin-system (RAS) halted the progression, or even induced a regression in renal injury. We studied the potential of the angiotensin-converting enzyme (ACE) inhibitor perindopril and the angiotensin receptor blocker candesartan to reverse the established renal injury in the obese Zucker rat (OZR). Methods: Forty 4-week-old OZRs were uninephrectomized and fed a high-protein diet. After 16 weeks, they were randomized into 4 groups $(n=10$ each) with comparable proteinuria: (1) control group sacrificed immediately for baseline data, and groups gavaged daily for 8 weeks with (2) placebo, (3) perindopril (1 mg/kg/ day), or (4) candesartan (10 mg/kg/day). Results: Both drugs reduced systolic blood pressure (perindopril -16\%, $\mathrm{p}<0.001$; candesartan $-10 \%, p<0.05)$, renal hypertrophy, and proteinuria (perindopril to $32 \%$; candesartan to $37 \%$ of pretreatment values). Glomerulosclerosis was halted (perindopril $p<0.001$; candesartan $p<0.05$ ), and the numbers of glo-
\end{abstract}

merular endothelial and podocyte cells were restored. Mesangiolysis was reversed by perindopril. Metabolic and oxidative parameters were either stabilized (perindopril), or improved (candesartan). Conclusion: In the OZR late inhibition of RAS halts the progression of glomerulosclerosis, reverses mesangiolysis and prevents the decline in glomerular endothelial cell and podocyte numbers. Tubulointerstitial fibrosis and vascular injury remain unchanged. Proteinuria shows marked regression.

Copyright $\odot 2008$ S. Karger AG, Basel

\section{Introduction}

Nephropathy due to type 2 diabetes mellitus is the leading cause of end-stage renal disease. A key factor in the progression of diabetic and non-diabetic renal disease is the renin-angiotensin system (RAS). In humans, or rodent models, angiotensin-converting enzyme (ACE) inhibitors (ACEIs) and angiotensin II (AngII) type 1 receptor blockers (ARBs) are effective in the prevention and control of renal damage. In experimental settings inhibitors of RAS even reversed the established non-diabetic renal injury [1-3], while pancreatic transplantation in humans reversed diabetic nephropathy (DN) [4].

\section{KARGER}

Fax +41613061234 E-Mail karger@karger.ch www.karger.com

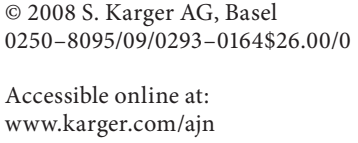

MU Dr. Katarína Šebeková

Slovak Medical University

Limbová 14

SK-833 03 Bratislava (Slovakia)

Tel. +421 259369 431, Fax +421 259369 170, E-Mail katarina.sebekova@szu.sk 


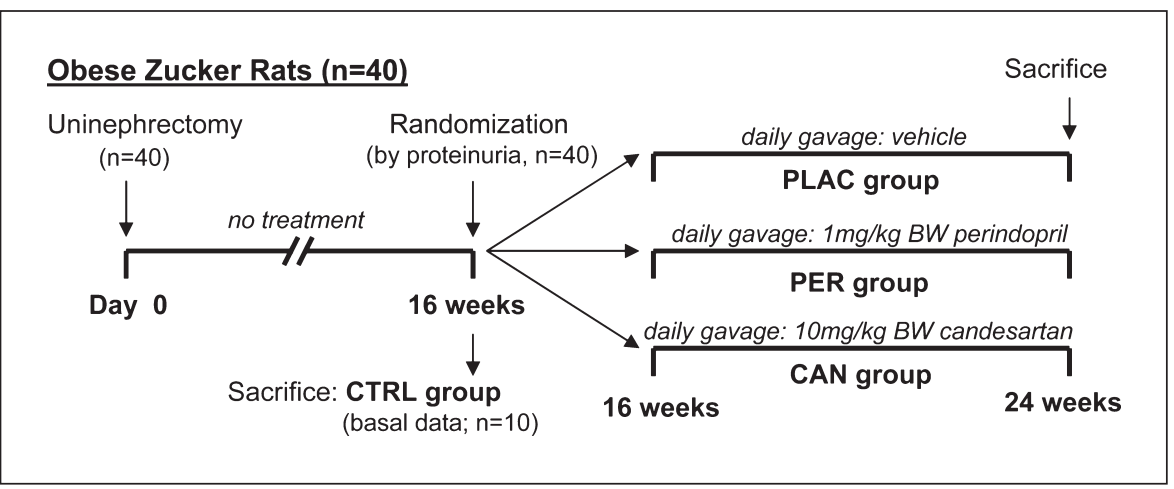

Fig. 1. The experimental protocol.

Obese Zucker rats (OZRs, a model of type 2 diabetes mellitus), develop hyperphagia, obesity, hyperglycemia, hyperinsulinemia, hyperlipidemia, hyperaggregation of platelets, moderate hypertension, and (at approximately 40 weeks of age) renal damage [5]. The effects of ACEIs, $\mathrm{ARB}$ and their combination in preventing renal injury in OZRs have already been described [6], but not evaluated in established DN. We show that 8-week treatment with the ACEI perindopril and the ARB candesartan, initiated in established $\mathrm{DN}$, ameliorates and even reverses functional and several aspects of glomerular kidney damage.

\section{Materials and Methods}

\section{Animals}

The research protocol was approved by the Ethics Committee for Animals (Bratislava, Slovakia). Forty 1-month-old male OZRs (Iffa Credo, Lyon, France) were subjected to unilateral nephrectomy under intraperitoneal ketamin/xylazin anesthesia [7]. Animals were housed under standard conditions with free access to water. After uninephrectomy, rats were switched from standard chow to a high-protein diet (50\%, Altromin, Lage, Germany). Four months later they were randomized into 4 groups (10 animals/group) with comparable proteinuria (fig. 1). Control rats were sacrificed before any treatment was initiated. By gavage, placebo rats received a $1 \%$ water solution of hydroxyethyl cellulose (HEC) in a volume of $5 \mathrm{ml} / \mathrm{kg}$. Perindopril (Servier, Paris, France) was administered at a dose of $1 \mathrm{mg} / \mathrm{kg} / \mathrm{day}$, and candesartan cilexetil (Astra Zeneca, Wedel, Germany) at a dose of $10 \mathrm{mg} / \mathrm{kg} / \mathrm{day}$, dissolved in 1\% HEC, 7 days/week for 8 weeks.

Rats were weighed weekly. Before sacrifice, systolic blood pressure (SBP) was measured by tail phethysmography. Twentyfour-hour urine was collected in metabolic cages before randomization and sacrifice. Blood was sampled under anesthesia from the abdominal aorta.

\section{Biochemical Analyses}

Routine blood and urine biochemistry (Vitros 250 Analyzer; J\&J, Rochester, Minn., USA) and blood count (Sysmex K-21 Ana- lyzer; Kobe, Japan) were analyzed. In plasma, the immunoreactive insulin concentration (Linco Research, St. Charles, Mo., USA) and advanced oxidation protein products (AOPPs) [8], and in erythrocytes glutathione peroxidase (GPX) activity (Randox Laboratories, Crumlin, UK) were determined.

\section{Renal Histology}

After retrograde perfusion fixation with glutaraldehyde via the abdominal aorta, kidneys were weighed and dissected into 1$\mathrm{mm}$-wide slices in a plane perpendicular to the interpolar axis [7]. Ten pieces/kidney were embedded in Epon-Araldite. Semithin (1$\mu \mathrm{m})$ slices were stained with methylene blue and basic fuchsin. Four-micrometer sections embedded in paraffin were stained with hematoxylin/eosin (HE) and periodic acid-Schiff (PAS). All morphological evaluations were performed in a blinded manner.

\section{Morphological Investigations}

Semiquantitative

Mesangiolysis (a hallmark of early glomerular damage in DN) was determined utilizing a $0-4$ scoring system $(0=$ normal to $4=$ severe damage) in 100 glomeruli/animal [7].

The degree of mesangial matrix expansion and sclerosis within the glomerular tuft (an index of progression) was determined on PAS-stained sections using a semiquantitative scoring system from 0 to $4(0=$ normal glomerulus, $4=$ severe damage $)$ in 100 glomeruli/animal $[7,9]$.

Tubulointerstitial and vascular damage indices were assessed on PAS-stained paraffin sections using the previously described scoring system $[7,9]$.

Glomerular Geometry

The volume density of glomeruli and the area density of glomerular tufts were measured by point counting on HE sections $[7,9]$. The total number of glomeruli was derived from the total volume of the renal cortex and the number of glomeruli per cortex volume. The mean glomerular tuft volume was determined.

Semithin sections were qualitatively inspected for glomerular cellular changes (podocyte hypertrophy and degeneration, and mesangial or endothelial cell hyperplasia). Five sections/animal (at least 30 glomeruli) were analyzed quantitatively for glomerular cell number and volume determination [9]. 
Table 1. Animal data, blood and urine chemistry

\begin{tabular}{|c|c|c|c|c|c|c|}
\hline & \multirow[t]{2}{*}{ CTRL } & \multirow[t]{2}{*}{ PLAC } & \multirow[t]{2}{*}{ PER } & \multirow[t]{2}{*}{ CAN } & \multicolumn{2}{|c|}{ ANOVA } \\
\hline & & & & & $\mathrm{F}$ & $\mathrm{p}$ \\
\hline Body weight $t 0, g$ & $524 \pm 32$ & $542 \pm 52$ & $526 \pm 41$ & $508 \pm 40$ & 1.57 & 0.223 \\
\hline Body weight $t 8, g$ & $\mathrm{ND}$ & $543 \pm 64$ & $493 \pm 62$ & $476 \pm 52$ & 3.26 & 0.055 \\
\hline Kidney weight, g & $2.2 \pm 0.3$ & $3.4 \pm 0.9^{++}$ & $2.6 \pm 0.5^{*}$ & $2.3 \pm 0.3^{* * *}$ & 6.63 & 0.005 \\
\hline Kidney/body weight, mg/g & $4.3 \pm 0.5$ & $6.1 \pm 1.2^{++}$ & $5.2 \pm 1.0$ & $4.9 \pm 0.2^{*}$ & 4.50 & 0.021 \\
\hline $\mathrm{SBP}, \mathrm{mm} \mathrm{Hg}$ & $\mathrm{ND}$ & $147 \pm 17$ & $124 \pm 12^{* * *}$ & $133 \pm 11^{*}$ & 7.10 & 0.004 \\
\hline Creatinine, $\mu \mathrm{mol} / \mathrm{l}$ & $53 \pm 24$ & $64 \pm 16$ & $74 \pm 13$ & $74 \pm 17$ & 1.28 & 0.295 \\
\hline $\mathrm{Cl}_{\text {Crea }}, \mathrm{ml} / \mathrm{min}$ & $0.9 \pm 0.4$ & $0.8 \pm 0.2$ & $0.6 \pm 0.1$ & $0.7 \pm 0.2$ & 1.82 & 0.184 \\
\hline Glucose, $\mathrm{mmol} / \mathrm{l}$ & $28 \pm 9$ & $23 \pm 3$ & $24 \pm 5$ & $24 \pm 7$ & 0.13 & 0.878 \\
\hline IRI, ng/ml & $1.0 \pm 0.9$ & $2.2 \pm 1.0^{+}$ & $2.1 \pm 0.8$ & $1.3 \pm 0.4^{*}$ & 3.60 & 0.042 \\
\hline Cholesterol, mmol/l & $7.3 \pm 2.0$ & $6.7 \pm 1.4$ & $6.5 \pm 1.0$ & $6.2 \pm 1.1$ & 0.45 & 0.645 \\
\hline Triglycerides, $\mathrm{mmol} / \mathrm{l}$ & $2.2 \pm 0.4$ & $2.1 \pm 0.7$ & $2.6 \pm 0.9$ & $1.3 \pm 0.7$ & 6.58 & 0.005 \\
\hline Leukocyte count, $\times 10^{9} / \mu \mathrm{l}$ & $4.6 \pm 1.4$ & $8.8 \pm 5.2^{+}$ & $5.2 \pm 2.8$ & $6.8 \pm 4.5$ & 1.73 & 0.198 \\
\hline AOPPs $/ \mathrm{Alb}, \mu \mathrm{mol} / \mathrm{g}$ & $2.2 \pm 0.6$ & $1.7 \pm 0.6$ & $1.6 \pm 0.5$ & $0.8 \pm 0.6^{* * *}$ & 7.28 & 0.003 \\
\hline GPX activity, U/g Hb & $29.5 \pm 5.7$ & $25.0 \pm 3.1^{+}$ & $28.5 \pm 4.0$ & $28.5 \pm 4.3$ & 2.43 & 0.109 \\
\hline
\end{tabular}

CTRL $=$ Control obese Zucker rats sacrificed before initiation of the treatment; $\mathrm{PLAC}=$ placebo $\mathrm{PER}=$ perindopril; $\mathrm{CAN}=$ candesartan; $\mathrm{t} 0$ = before initiation of treatment; $\mathrm{t} 8=$ after 8 weeks of treatment; $\mathrm{SBP}=$ systolic blood pressure; $\mathrm{Cl}_{\text {crea }}=$ creatinine clearance; IRI = immunoreactive insulin; AOPPs = advanced oxida- tion protein products; $\mathrm{Alb}=$ albumin; $\mathrm{GPX}=$ glutathione peroxidase; $\mathrm{Hb}=$ hemoglobin; $\mathrm{ND}=$ not determined (technical reasons). PLAC.

\section{Statistics}

Data are expressed as mean \pm SD. Control and placebo groups were compared employing Student's t test or the Mann-Whitney U test, where appropriate. One-way ANOVA with Dunnett's posthoc test was used to compare placebo and treated groups. Twoway ANOVA with Duncan's post-hoc test was used to analyze the data on diuresis and proteinuria. A $p$ value of $<0.05$ was considered significant.

\section{Results}

\section{Body Weight, Kidney Weight and SBP (table 1)}

During treatment, the body weight of the placebo rats remained stable, while it tended to decrease in the treated groups. Compared to placebo, perindopril- and candesartan-treated rats had lower SBP by $16 \%(-23 \mathrm{~mm} \mathrm{Hg}$; $\mathrm{p}<0.001)$ and $10 \%(-14 \mathrm{~mm} \mathrm{Hg}$; $\mathrm{p}<0.05)$, respectively. Kidney weight was higher in placebo rats $(+55 \%, \mathrm{p}<0.01$ vs. controls). Both treatments effectively halted hypertrophy, but after correction for body weight it remained significant only in the candesartan group (vs. placebo, $\mathrm{p}<0.05)$.

\section{Renal Function (table 1)}

An insignificant rise in mean plasma creatinine (21\%), and a decreased creatinine clearance $(-12 \%$; n.s.) were observed in placebo rats (vs. controls). Both treated groups had an insignificantly higher plasma creatinine concentration ( $+16 \%$ vs. placebo), and lower creatinine clearance (perindopril -33\%; candesartan -14\%; n.s.).

At treatment initiation, groups had comparable diuresis and proteinuria (fig. 2, 3). After treatment, diuresis increased. In the placebo group proteinuria increased (+21\%; n.s.), perindopril and candesartan lowered proteinuria to $32(\mathrm{p}<0.01)$ and $37 \%(\mathrm{p}<0.001)$ of pretreatment values, respectively.

\section{Kidney Morphology (fig. 4, 5; table 2)}

$\mathrm{DN}$ in placebo rats markedly progressed during the treatment period: mesangiolysis $(+100 \%)$; glomerulosclerosis (+33\%); tubulointerstitial fibrosis $(+56 \%)$, and vascular damage scores $(+61 \%)$ increased (all p < 0.001). Candesartan stopped the progression of mesangiolysis, while perindopril reversed it $(-32 \%)$. Both treatments halted the progression of glomerulosclerosis. Mild tubulointerstitial or vascular damage remained unaffected.

Placebo rats developed glomerular hypertrophy; perindopril slightly ameliorated it ( $-11 \%$ vs. placebo; n.s.). 


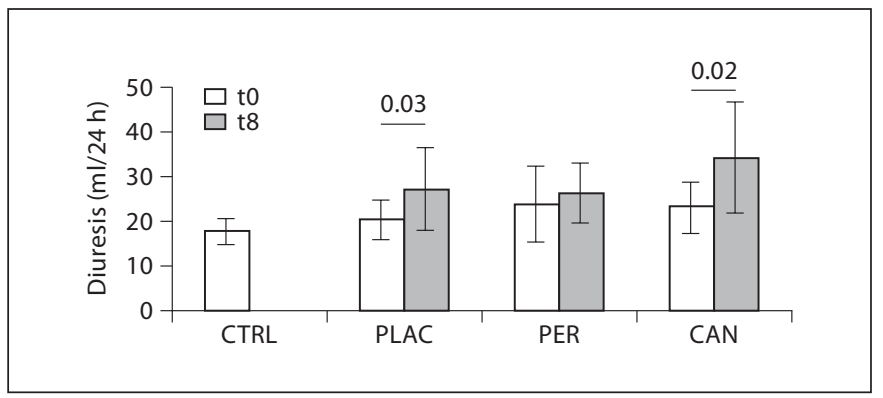

Fig. 2. Time course of diuresis. CTRL = Controls; PLAC $=$ placebo; $\mathrm{PER}=$ perindopril; $\mathrm{CAN}=$ candesartan; $\mathrm{t} 0=$ before the treatment; $\mathrm{t} 8=$ after 8 weeks of treatment.

The total number of glomerular cells did not differ significantly between placebo and control rats. In both treatment groups endothelial cell and podocyte numbers were significantly higher than in the placebo group. In parallel, with a trend towards lower endothelial cell number, glomerular capillary length density (as a three-dimensional parameter of glomerular capillarization) was significantly lower in the placebo group vs. the controls. Both treatments had no significant effect on glomerular capillarization.

\section{Blood Chemistry (table 1)}

All rats were hyperglycemic. Placebo rats had higher insulinemia than the controls, or candesartan-administered animals. All groups showed dyslipidemia, which was not significantly reduced by the treatments. Placebo rats had significantly higher leukocyte count (vs. controls), which was insignificantly reduced by both treatments.

\section{Oxidative Status (table 1)}

Plasma AOPPs did not differ significantly between the control and placebo groups. Candesartan reduced AOPP levels $(-38 \%$ vs. placebo; $p<0.01)$. GPX activity was significantly lower in the placebo rats (vs. the controls), and both treatments only partially restored it.

\section{Discussion}

To date, no data are available on the secondary prevention of renal injury in the OZRs by blockade of the RAS. The major finding of this study shows that the RAS blockade in established DN in OZRs, either with perindopril

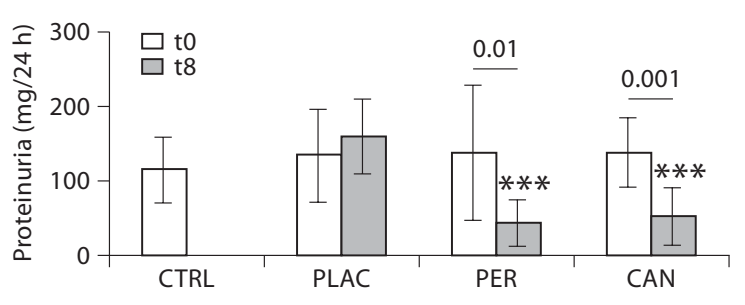

Fig. 3. Time course of proteinuria. $\mathrm{CTRL}=$ Controls; $\mathrm{PLAC}=$ placebo; $\mathrm{PER}=$ perindopril; $\mathrm{CAN}=$ candesartan; $\mathrm{t} 0=$ before the treatment; $\mathrm{t} 8=\operatorname{after} 8$ weeks of treatment. ${ }^{* *} \mathrm{p}<0.001$ vs. PLAC.

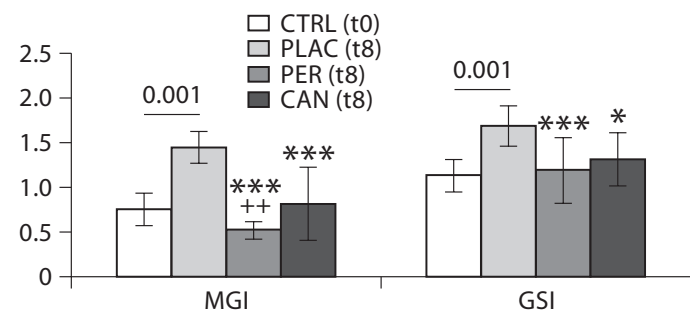

Fig. 4. Renal damage scores. $\mathrm{CTRL}=$ Controls; $\mathrm{PLAC}=$ placebo; $\mathrm{PER}=$ perindopril $\mathrm{CAN}=$ candesartan; $\mathrm{t} 0=$ before the treatment; t8 = after 8 weeks of treatment; MGI = Mesangiolysis index; GSI $=$ glomerulosclerosis index. ${ }^{*} \mathrm{p}<0.05,{ }^{* * *} \mathrm{p}<0.001 \mathrm{vs.} \mathrm{PLAC;}$ ${ }^{++} \mathrm{p}<0.01$ vs. CTRL.

or candesartan, not only halts the progression of several markers of renal injury, but even induces a partial regression of glomerular damage. Both treatments retarded the progression of glomerulosclerosis and preserved glomerular cells (podocytes, endothelial cells, glomerular capillary supply). Candesartan halted and perindopril induced a limited regression of mesangiolysis (in contrast to other studies) [6]. Tubulointerstitial and vascular sclerosis scores were not significantly affected. Both treatments suppressed proteinuria. The missing effect on the extraglomerular structures may reflect the persisting risk factors of renal damage, e.g. hyperglycemia, hypertension, dyslipidemia, obesity, and high protein intake. Moreover, in the OZRs the systemic RAS is downregulated, limiting the effect of ACE inhibition/blockade [10]. 

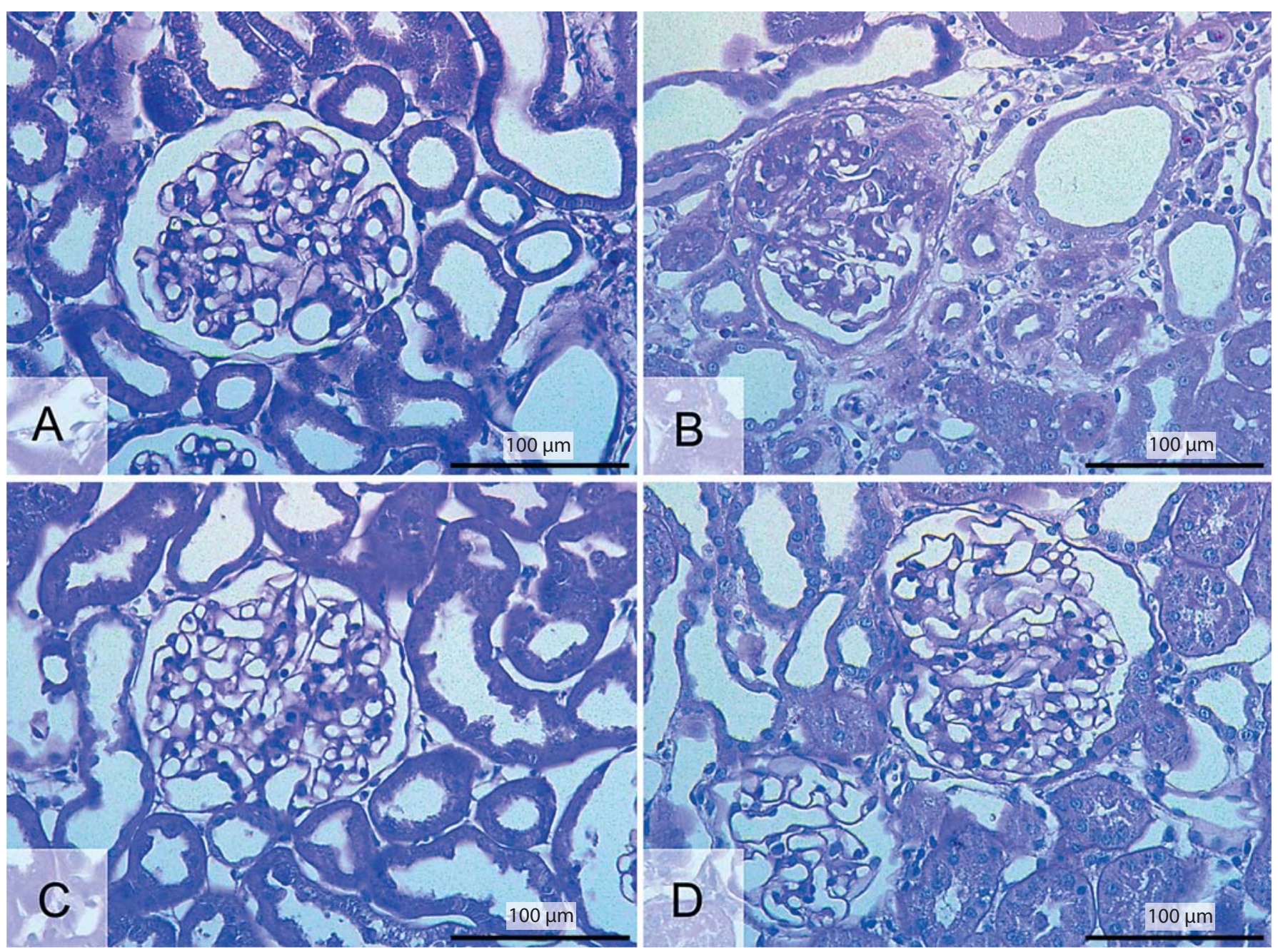

Fig. 5. Representative changes in glomerular morphology. Paraffin sections. A Glomerulus of a uninephrectomized OZR after a 4-month-long period without treatment (basal data). B Glomerulus from placebo-administered OZR showing irregular structures

of the capillary tuft with capillary widening, capillary dilatation and dissolution of the mesangium (mesangiolysis score 1-2). C, D Glomerulus of perindopril- (C) and candesartan- (D) treated OZR with a nearly normal capillary structure and mesangium.

A major mechanism of renoprotection under RAS blockade is the antihypertensive effect, as in our study. However, the decline in blood pressure seems to be too mild to explain the marked improvement in renal injury, thus other mechanisms have to be considered.

\section{Oxidative Stress}

AngII promotes generation of reactive oxygen species (ROS) which worsen insulin resistance, diabetes, and its complications [11]. AOPPs reflect, and trigger, an oxidative burst of phagocytes [8]. In our study, candesartan lowered AOPP levels, similar to peritoneal dialysis patients [12]. In line with the increased AOPPs, the placebo

rats had a higher leukocyte count which, even within the normal range, is associated with macro- and microvascular complications in diabetics [13]. While perindopril increased the activities of antioxidant enzymes in hypertensive patients [14], decreased GPX activity in our placebo rats was only partially restored under both treatments. Thus, other antioxidative mechanisms could have been involved.

\section{Carbohydrate/Lipid Metabolism}

ARB administration decreases peripheral insulin resistance in type 2 diabetics and animal models [15]. We observed the insulin-sensitizing effects of candesartan, 
Table 2. Renal histomorphology

\begin{tabular}{|c|c|c|c|c|c|c|}
\hline & \multirow[t]{2}{*}{ CTRL } & \multirow[t]{2}{*}{ PLAC } & \multirow[t]{2}{*}{ PER } & \multirow[t]{2}{*}{ CAN } & \multicolumn{2}{|c|}{ ANOVA } \\
\hline & & & & & $\mathrm{F}$ & $\mathrm{p}$ \\
\hline TSI & $0.14 \pm 0.06$ & $0.32 \pm 0.08^{+++}$ & $0.28 \pm 0.05$ & $0.25 \pm 0.07$ & 2.60 & 0.094 \\
\hline VSI & $0.11 \pm 0.03$ & $0.28 \pm 0.07^{+++}$ & $0.33 \pm 0.07$ & $0.28 \pm 0.09$ & 1.38 & 0.269 \\
\hline Glomerular volume, $\mu \mathrm{m}^{3} \cdot 10^{3}$ & $629 \pm 36$ & $705 \pm 50^{++}$ & $649 \pm 24$ & $688 \pm 55$ & 3.39 & 0.050 \\
\hline Length density of glomerular capillaries, $\mathrm{mm} / \mathrm{mm}^{3}$ & $8,599 \pm 509$ & $7,543 \pm 548^{+++}$ & $7,319 \pm 882$ & $8,181 \pm 684$ & 3.52 & 0.046 \\
\hline Podocyte number & $110 \pm 23$ & $98 \pm 16$ & $129 \pm 21^{* * *}$ & $121 \pm 16^{*}$ & 7.63 & 0.003 \\
\hline Mesangial cell number & $215 \pm 36$ & $249 \pm 50$ & $244 \pm 52$ & $273 \pm 52$ & 0.823 & 0.451 \\
\hline Endothelial cell number & $380 \pm 51$ & $333 \pm 47$ & $410 \pm 63^{* * *}$ & $443 \pm 48^{* * *}$ & 10.89 & 0.001 \\
\hline Parietal cell number & $199 \pm 22$ & $218 \pm 21$ & $226 \pm 13$ & $230 \pm 36$ & 0.55 & 0.582 \\
\hline Total number of glomerular cells & $904 \pm 73$ & $898 \pm 99$ & $1,009 \pm 128$ & $1,067 \pm 102^{* * *}$ & 5.84 & 0.008 \\
\hline
\end{tabular}

$\mathrm{CTRL}=$ Control obese Zucker rats sacrificed before initiation of treatment $\mathrm{PLAC}=$ placebo $\mathrm{PER}=$ perindopril; $\mathrm{CAN}=\mathrm{candesar}-$ tan; TSI = index of tubulointerstitial damage; VSI = index of vascular damage.

${ }^{++} \mathrm{p}<0.01,{ }^{+++} \mathrm{p}<0.001$ vs. CTRL; ${ }^{*} \mathrm{p}<0.05,{ }^{* * *} \mathrm{p}<0.001$ vs. PLAC.

similar to a study in OZRs administered irbesartan [16]. Neither treatment influenced dyslipidemia, despite the reduction in proteinuria. In other studies candesartan either lowered or induced mild changes in plasma lipids $[17,18]$. Although perindopril did not significantly affect metabolic parameters, in hypertensive type 2 diabetics it improved some metabolic parameters compared with candesartan [18].

\section{Fat Tissue}

Obesity is a risk factor for progression of renal disease [19]. Adipocytes produce all components of the RAS [20]. RAS blockade in rodents lowered body weight gain, fat mass, adipocyte size and $\mathrm{AT}_{1}$ receptor number [21]. Thus, the decline in body weight in both treatment groups could be another protective mechanism of RAS blockade. However, in clinical studies, lowering of body weight under RAS blockade has not been reported.

\section{Renal Mechanisms}

In line with previous reports $[3,22,23]$, preservation of podocytes, glomerular endothelial cells, and reduced mesangial sclerosis were important renoprotective effects of RAS blockade also in established DN in OZRs. As vascular endothelial growth factor (VEGF) is considered a key downstream mechanism, the preservation of endothelial cells and podocytes could have been mediated via VEGF. Perindopril resulted in higher capillary density and VEGF synthesis in the myocardium of OZRs [24], and in the ischemic hindlimb of diabetic mice [25].
The lowered glomerular filtration rate in the treated groups is most probably functional, partly via decreased systemic blood pressure, as is well known after ACEI administration [26].

Since single ACEI and ARB compounds differ in their pharmacological properties, we cannot conclude whether our data represent a class effect or merely that of the drugs employed. It is unclear whether RAS blockade could reverse $\mathrm{DN}$ also in humans.

In conclusion, the late intervention with perindopril and candesartan in established DN in uninephrectomized OZRs halted the progression and even induced a partial regression in glomerular injury, preserving glomerular cells and capillaries. The blood pressure-lowering actions of RAS blockade as well as additional effects (reduction of oxidative stress, improved carbohydrate metabolism) are to be considered, in particular for candesartan.

\section{Acknowledgements}

The study was supported in part by the Institut de Recherches Internationales Servier, Courbevoie, France, by the Deutsche Forschungsgemeinschaft (DFG, SFB423, project B8/Z2) and by the Verein zur Bekämpfung der Hochdruck- und Nierenkrankheiten Würzburg e.V., Germany. The technical assistance of Monika Klewer and Miriam Ramming is gratefully acknowledged. 


\section{References}

1 Boffa JJ, Lu Y, Placier S, et al: Regression of renal vascular and glomerular fibrosis: role of angiotensin II receptor antagonism and matrix metalloproteinases. J Am Soc Nephrol 2003;14:1132-1144.

2 Remuzzi A, Gagliardini E, Sangalli F, et al: ACE inhibition reduces glomerulosclerosis and regenerates glomerular tissue in a model of progressive renal disease. Kidney Int 2006; 69:1124-1130.

3 Adamczak M, Gross ML, Krtil J, et al: Reversal of glomerulosclerosis after high-dose enalapril treatment in subtotally nephrectomized rats. J Am Soc Nephrol 2003; 14:28332842.

4 Fioretto P, Steffes MW, Sutherland DE, et al: Reversal of lesions of diabetic nephropathy after pancreas transplantation. N Engl J Med 1998;339:69-75.

5 Kasiske BL, O'Donnell MP, Keane WF: The Zucker rat model of obesity, insulin resistance, hyperlipidemia and renal injury. Hypertension 1992;19(suppl 1):I110-I115.

6 Toblli JE, DeRosa G, Cao G, et al: ACE inhibitor and angiotensin type I receptor antagonist in combination reduce renal damage in obese Zucker rats. Kidney Int 2004;65: 2343-2359.

7 Šebeková K, Eifert T, Klassen A, et al: Renal effects of S18886 (terutroban), a TP receptor antagonist, in an experimental model of type 2 diabetes. Diabetes 2007;56:968-974.

8 Witko-Sarsat V, Friedlander M, Nguyen Khoa $\mathrm{T}$, et al: Advanced oxidation protein products as novel mediators of inflammation and monocyte activation in chronic renal failure. J Immunol 1998; 161:2524-2532.

9 Gross ML, Ritz E, Schoof A, et al: Renal damage in the SHR/N-cp type 2 diabetes model: comparison of an angiotensin-converting enzyme inhibitor and endothelin receptor blocker. Lab Invest 2003;83:1267-1277.
10 Harker CT, O'Donnell MP, Kasiske BL, et al: The renin-angiotensin system in the type II diabetic obese Zucker rat. J Am Soc Nephrol 1993;6:1354-1361.

11 Evans JL, Maddux BA, Goldfine ID: The molecular basis for oxidative stress-induced insulin resistance. Antioxid Redox Signal 2005; 7:1040-1052.

12 Furuya R, Odamaki M, Kumagai H, et al: Impact of angiotensin II receptor blocker on plasma levels of adiponectin and advanced oxidation protein products in peritoneal dialysis patients. Blood Purif 2006;24:445450.

13 Tong PC, Lee KF, So WY, et al: White blood cell count is associated with macro- and $\mathrm{mi}$ crovascular complications in Chinese patients with type 2 diabetes. Diabetes Care 2004;27:216-222.

14 Kedziora-Kornatowska K, Kornatowski T, Bartosz G, et al: Production of nitric oxide, lipid peroxidation and oxidase activity of ceruloplasmin in blood of elderly patients with primary hypertension. Effects of perindopril treatment. Aging Clin Exp Res 2006;18:1-6.

15 Jandeleit-Dahm KA, Tikellis C, Reid CM, et al: Why blockade of the renin-angiotensin system reduces the incidence of new-onset diabetes. J Hypertens 2005;23:463-473.

16 Munoz MC, Argentino DP, Dominici FP, et al: Irbesartan restores the in-vivo insulin signaling pathway leading to Akt activation in obese Zucker rats. J Hypertens 2006;24 1607-1617.

17 Noda M, Matsuo T, Nagano-Tsuge $H$, et al: Involvement of angiotensin II in progression of renal injury in rats with genetic non-insulin-dependent diabetes mellitus (Wistar fatty rats). Jpn J Pharmacol 2001;85:416-422.
18 Derosa G, Cicero AF, Ciccarelli L, et al: A randomized, double-blind, controlled, parallel-group comparison of perindopril and candesartan in hypertensive patients with type 2 diabetes mellitus. Clin Ther 2003;25: 2006-2021.

19 Abrass CK: Overview: obesity: what does it have to do with kidney disease? J Am Soc Nephrol 2004;15:2768-2772.

20 Pintérova L, Križanová O, Zorád S: Rat epididymal fat tissue express all components of the renin-angiotensin system. Gen Physiol Biophys 2000;19:329-334

21 Furuhashi $M$, Ura $N$, Takizawa $H$, et al: Blockade of renin-angiotensin system decreases adipocyte size with improvement of insulin sensitivity. J Hypertens 2004;22: 1977-1982.

22 Liang XB, Ma LJ, Naito T, et al: Angiotensin type 1 receptor blocker restores podocyte potential to promote glomerular endothelial cell growth. J Am Soc Nephrol 2006;217: 1886-1895.

23 Blanco S, Bonet J, Lopez D, et al: ACE inhibitors improve nephrin expression in Zucker rats with glomerulosclerosis. Kidney Int 2005;93:10-14.

24 Toblli JE, Cao G, DeRosa G, et al: Angiotensin-converting enzyme inhibition and angiogenesis in myocardium of obese Zucker rats. Am J Hypertens 2004;17:172-180.

25 Ebrahimian TG, Tamarat R, Clergue M, et al: Dual effect of angiotensin-converting enzyme inhibition on angiogenesis in type 1 diabetic mice. Arterioscler Thromb Vasc Biol 2005;25:65-70

26 Bakris GL, Weir MR: Angiotensin-converting enzyme inhibitor-associated elevations in serum creatinine: is this a cause for concern? Arch Intern Med 2000;160:685-693. 